\title{
THE ROLE OF PHARMACISTS IN EVALUATING AND INTERVENING THE PATIENTS WITH DIABETIC NEUROPATHY
}

\author{
SESILIA ANDRIANI KEBAN ${ }^{1 *}$, NAJUAH NAJUAH ${ }^{2}$, SYAMSUDIN ABDILLAH ${ }^{1}$
}

\begin{abstract}
${ }^{1}$ Department of Clinical and Community Pharmacy, Faculty of Pharmacy, Pancasila University, Jl. Srengseng Sawah, Jagakarsa, South Jakarta, Indonesia. ${ }^{2}$ Postgraduate of Hospital Pharmacy, Faculty of Pharmacy, Pancasila University, Jakarta, Indonesia.
\end{abstract} Email: sesil.ffup@gmail.com

Received: 20 August 2016, Revised and Accepted: 27 October 2016

\section{ABSTRACT}

Objective: The purpose of this study was to estimate the prevalence and risk factors for diabetic peripheral neuropathy, to evaluate the score of neuropathy, and to determine the effect of pharmacist intervention toward diabetic neuropathy (DN) patients at Gatot Soebroto Hospital, Jakarta, in 2013

Methods: An analytic research was conducted using cross-sectional approach to find out the effect of pharmacist intervention toward DN risk factors and prevalence. Toronto clinical scoring system was used to score the symptoms and physical examination results. Data about sociodemographic characters, age, duration of diabetic, blood glucose, blood pressure, cardiovascular diseases, lifestyle, body mass index (BMI), and smoking were collected. Pharmacist intervention was given to increase patient information about DN and its risks factors.

Results: There were 59 respondents involved in this study. It can be found that $15.3 \%$ respondents had mild diabetic peripheral neuropathy, $1.7 \%$ had moderate diabetic peripheral neuropathy, $1.7 \%$ had severe diabetic peripheral neuropathy, and as much as $81,4 \%$ respondents had no neuropathy. There was a correlation (but not statistically significant) between diabetic peripheral neuropathy and its' risks factors such as ages, duration of diabetes, sex, cardiovascular disease (hypertension and cardiac disease), and lifestyle (smoking habit and BMI).

Conclusion: Pharmacist intervention showed an increase on the patient's knowledge about DN and also a significant decrease on the patient's blood glucose level $(\mathrm{p}<0.05)$.

Keywords: Diabetic neuropathy, Pharmacist role, Prevalence, Score, Risk factors, Blood glucose.

(C) 2017 The Authors. Published by Innovare Academic Sciences Pvt Ltd. This is an open access article under the CC BY license (http://creativecommons. org/licenses/by/4. 0/) DOI: http://dx.doi.org/10.22159/ajpcr.2017.v10i2.14796

\section{INTRODUCTION}

Diabetes mellitus (DM) is a metabolic disorder, which is characterized by hyperglycemia [1]. The prevalence keeps increasing with time. In 1995 , it was oleh $4.0 \%$, and in 2015 , it is predicted to reach a level of $5.4 \%$. In 1995, there were around 135 million of diabetes mellitus cases, and in 2015, it is predicted to increase to 300 million of cases [2].

The 2012 report of patients' data at the Internal Department of Gatot Soebroto Hospital, Jakarta, showed that diabetes mellitus ranked the first with 14.508 cases, followed by hypertension with 5.875 cases and dyspepsia syndrome with 1.008 cases. Diabetes mellitus is a chronic disease that needs continuous treatment and education for the patients to prevent acute complication and to reduce the risk of longterm complication [3]. Long-term complication from diabetes includes retinopathy, which can lead to vision loss, nephropathy that leads to renal failure, peripheral neuropathy followed by the risks of foot ulcer, amputation, and charcot joins, as well as autonomous neuropathy that leads to disorders in the gastrointestinal and urogenital tracts, cardiovascular symptoms, and erectile dysfunction [1].

Diabetic neuropathy (DN) is the presence and/or signs of peripheral nervous dysfunctions in patients with diabetes after the exclusion of other causes. About $60-70 \%$ of diabetic patients suffer from moderate to severe nervous system damage [4]. Diabetic peripheral neuropathy occurs in $50 \%$ of diabetic patients [5]. Duration of the disease is one of the factors that lead to increasing morbidity and mortality rates [6]. The prevalence of DN is $11.1 \%$ in the population at the age of $23-40$ ages. It increased to 32.3 years in the patients at the age of $60-80$ years. When it comes to duration, $14.1 \%$ of the patients who had diabetes for $<5$ years developed neuropathy and $29.2 \%$ of diabetic patients who have diabetes for 9-11 years developed neuropathy [7]. Another research even found that $73.9 \%$ of the patients who had diabetes for $<5$ years had neuropathy and $100 \%$ of the patients who have diabetes for $>15$ years had neuropathy [8]. Almost $30 \%$ of the diabetic patients at the age of 40 years or more had sensational impairment on legs, which is the severest form of DN and the main contributor for amputation. More than $60 \%$ of non-traumatic amputations occurred in diabetic patients [4]. Patients with diabetes mellitus and peripheral neuropathy were at increased risk for developing tissue damage, which was followed by diabetic feet, diabetic ulcers, and even foot amputation [9]. These situations are the major causes of morbidity and mortality. The incidence rate can be reduced when the peripheral neuropathy can be detected at the earliest stadium possible [5].

Risk factors for diabetic peripheral neuropathy include improper blood sugar control, duration of the disease, hyperlipidemia, elevated albumin excretion, smoking habit, alcohol, low economic status, renal failure, and increased body mass index (BMI). Diabetic peripheral neuropathy is frequently associated with cardiovascular diseases, mortality, and microangiopathy $[5,10-12]$.

The examination is necessary to prevent and treat nerve damage as the first step in identifying the symptoms in the sensor and motor systems, which are caused by the DN, after the exclusion of other causes. Clinical evaluation includes foot examination for foot pain, numbness, tingling, weakness and ataxia, and upper limb, examination for sensory system, which includes pinprick, temperature, light touch, vibration, and position sense, and examinations for Achilles reflex, and Patella reflex using reflex hammer [13]. 
Gatot Soebroto Hospital is a military hospital located in Central Jakarta. It is a Type I hospital that deals with many diabetes mellitus cases. It even ranked the first of all diseases at the Internal Department of Gatot Soebroto Hospital, Jakarta. It was estimated that in 2012, 11-12\% of 14.508 patients were diabetes mellitus cases and it is equaled to 145 cases each month. Screening and treatment, which had been given for DN were limited to patients who had nervous impairment, while education provided by the pharmacists was in adequate, because of the limited number of staffs. Based on the elaboration above, a research is necessary to find out the prevalence of DN and risks factors for the diabetic patients to be judged as having DN. It is measured using Toronto clinical scoring system (TCSS) and in-depth interview to identify other diseases that may be developed by the patients, including cardiovascular diseases (coronary arterial diseases, triglycerides, and hypertension) and lifestyles, such as smoking habit. Then, the pharmacists provide education for the patients. The education includes providing valid information on the preventive measures, management, and proper regimen in the patients with DN. Therefore, some questions related to this research include the following:

1. What is the prevalence of DN at Gatot Soebroto Hospital?

2. What are the risk factors of DN?

3. What are the score of neuropathy in patients involved in this study?

4. What is the effect of pharmacist intervention toward DN patients at Gatot Soebroto Hospital, Jakarta, in 2013.

Based on the questions above, a research on "The Role of Pharmacists in Evaluating and Intervening the Patients with DN" was conducted.

\section{METHODS}

Materials

DN patients, medical records, and TCSS.

\section{Methodology}

An analytic research was conducted using cross-sectional approach to find out the prevalence of DN and risk factors. Data on the risk factors were collected from medical records of the patients and in-depth interviews (questionnaire). The tool used to determine the level of neuropathy was TCSS. Education was provided by the pharmacists by distributing brochures or providing information directly to the samples on the certain type of complication from diabetes mellitus, namely DN. The research involved qualitative (descriptive) and quantitative analyses using the statistical program.

\section{Locus and time}

The research was conducted in October 2013, at the outpatient room of endocrine metabolic internal polyclinic, Gatot Soebroto Hospital, Jakarta.

\section{Population}

Type 2 diabetes outpatient who visited the Endocrine Department/ Metabolic Internal Clinic at Gatot Soebroto Hospital, Jakarta, every month and met the inclusion criteria.

\section{Respondents and number of respondents}

Respondents of the research were patients with Type 2 diabetes mellitus that met the inclusion criteria. 59 patients served as the respondents for the research.

\section{Determination of respondents}

The respondents were selected using nonrandomize incidental sampling technique with prospective data collection.

\section{Research stages}

1. Selection of patients with Type 2 diabetes mellitus that met the inclusion criteria and had outpatient services at Endocrine Department/Metabolic Internal Clinic at Gatot Soebroto Hospital, Jakarta.
Inclusion criteria: All of the patients with Type 2 diabetes mellitus at the outpatient room of Gatot Soebroto Hospital, Jakarta, age of $>50$ years, duration of diabetes for $>5$ years, and willingness to participate in the research by filling up informed consent.

Exclusion criteria: Diabetic patients at the age of $<50$ years, duration of diabetes $<5$ years, patients with Type 2 diabetes mellitus with rheumatic, vitamin B12 deficiency, hypothyroidism, and drug abuse.

2. Filling up informed consent. The patients were given an explanation on the procedure, objective, and benefits of the research before filling up the informed consent.

3. Recording of complete identity of the patients in the data collection sheet, which included a number of medical record, name, age, and sex.

4. Recording of physical examination results (height, weight, blood pressure, and total cholesterol level) and laboratory examination from the medical record.

5. In-depth interviews to explore the risk factors for DN (blood glucose, hypertension, cholesterol, duration of diabetes, and smoking habit).

6. Examination by the physician for sensory function, motor function, and reflexes using TCSS. The examinations included: Examinations for the symptoms:

a. Interview on the neuropathic symptoms on lower and upper extremities: Pain, numbness, tingling, and weakness

b. Examination for ataxia (movement coordination)

Examinations for reflexes:

a. Examination for patella reflex on both feet

b. Examination for Achilles reflex on both feet Sensory examination:

a. Sensory examination that included pinprick using lancet needle

b. Examination of temperature using hot water at a temperature of $40-50^{\circ} \mathrm{C}$ and $10-20^{\circ} \mathrm{C}$

c. Examination of slow touch using cotton

d. Examination of vibration using a $128-\mathrm{Hz}$ tuning fork

e. Examination of position sense by passive moving of the toes f. Evaluation of TCSS

g. Education by the pharmacists. Before the education, the patients were given questionnaire on DN, followed by education session by providing brochures and information on the DN.

\section{RESULTS}

Sample of the research was 68 patients, who visited for outpatient services Endocrine Department/Metabolic Internal Clinic at Gatot Soebroto Hospital, Jakarta. During the research course, 9 patients dropped out because of failure to visit every month. Therefore, 59 patients were used as sample. The research was conducted for 3 months and 3 sessions of education were given to the patients with an interval of 1 month between the educations. Demographic data on the patient characteristic can be seen on Table 1 .

The total populations involved in the research were 59 respondents that consisted of the following: $35.6 \%$ of the respondents aged $50-59$ years, $32.2 \%$ aged $60-69$ years, and $32.3 \%$ age $70-80$ years. Based on the gender, $44.1 \%$ of the respondents were male and the remaining $55.9 \%$ were female. Based on duration of the disease, $54.2 \%$ had been exposed to diabetes mellitus for 5-9 years, $33.9 \%$ for $10-19$ years, $8.8 \%$ for 20-29 years, and $3.4 \%$ for $>30$ years. Based on smoking habit, $28.6 \%$ of the respondents were smoking and $81.4 \%$ were not. Most of the respondents had normal body weight $(67.8 \%)$ with $28.8 \%$ overweight and $3.4 \%$ obese; $54.2 \%$ of the respondents had hypertension and normal $45,8 \%$ were normotensive; $55,9 \%$ of the respondents had high cholesterol level and $44.1 \%$ had a normal level. Finally, $11.9 \%$ of the respondents had heart disease and $88.1 \%$ did not.

Table 2 shows that $81.4 \%$ of the respondents had no neuropathy, $15.3 \%$ had mild neuropathy, $1.7 \%$ had moderate neuropathy, and $1.7 \%$ had severe neuropathy. 
Table 3 showed the relationship between neuropathy and risk factors. It can be seen that the relationship between dependent variables of neuropathy and independent variable, namely DN, could be significant, with the Confidence Interval of $\mathrm{p}<0.05$. However, when $\mathrm{P}$ value is more than 0.05 , it means that there is no significant relationship between neuropathy and the independent variables. Table 3 shows that significance values of the dependent variables of neuropathy were all $>0.05$. However, there is still a correlation between the incidence of DN at Gatot Soebroto Hospital, Jakarta, with the risk factors for DN, namely, age, sex, duration of diabetes mellitus, blood glucose, hypertension, heart disease, cholesterol, smoking habit, and BMI.

From Table 4, it can be seen that the percent knowledge of the respondents with DN before the intervention was $54.5 \%$, compared to that of $68.2 \%$ after the first intervention, and $90.9 \%$ after the second intervention. Meanwhile, knowledge of samples without neuropathy was $61.5 \%$ before the intervention, $64.6 \%$ after the first intervention, and $90.6 \%$ after the second intervention. In this case, it was found that knowledge of the respondents with and without DN increased after the first and the second intervention.

The research also found that $72.7 \%$ of the DN respondents had knowledge on the signs and symptoms of DN before the intervention, and it increased to $86.4 \%$ after the first intervention and $86.4 \%$ after the second intervention. On the other hand, $79.2 \%$ of the samples without neuropathy had knowledge on the signs and symptoms of DN before the intervention and it increased to $86.5 \%$ after the first intervention and $92.7 \%$ after the second intervention.

\section{DISCUSSION}

The major morbidity caused in the diabetic patients is due to dysfunction of several organ systems after a few years of having diabetes. Research has now clearly proved that these organ dysfunctions have their roots in diabetic pathophysiology. Many components of this pathophysiology have been identified. These components work interdependently with each other. One of the important factors identified is inflammation, which embraces the mechanisms such as oxidative stress, neovascularization, apoptosis, and cellular proliferation. It has been shown that inflammation plays an important role in microvascular as well as macrovascular complications of diabetes [14].

Neuropathy is the most common complication in diabetic patients. ${ }^{3}$ Even though the estimated prevalence of DN varied greatly depending on the diagnostic criteria, at least $50 \%$ of diabetic patients had neuropathy and $30-50 \%$ of prediabetic patients had neuropathy. The severity of DN is related to duration of the disease and blood glucose level. In a study conducted in the patients who had been diagnosed with diabetes mellitus for 25 years, $12 \%$ of the patients had DN and the incidence increased to more than $50 \%$ after 25 years. An evaluation on 6.487 diabetic patients was conducted in the United Kingdom and it was found that $5 \%$ of the patient's age $20-29$ years had DN and it increased to $44.2 \%$ in the patients at the age group of $70-79$ years. Similarly, a report showed that $33 \%$ of patients at the age group of $18-70$ years had DN and it increased to more than $50 \%$ in the elderly group [6].

One of the complications from diabetes mellitus is nervous damage, which is called DN. Its signs include foot pain. The characteristics of neuropathy on the feet include burning sensation and pinprick, numbness, tingling, weakness, ataxia (movement coordination) in addition to decreased reflex, and sensory system. Sensory nerve damage may lead to inability to perceive pain, heat, coldness, and or soft touch. Early examination is essential to prevent and treat the nerve damage. The first phase may include finding out sensory and motor symptoms caused by diabetes mellitus. Clinical evaluation includes an examination of feet, Achilles reflex, and Patella reflex using a tuning fork or biothesiometer [15].

TCSS was used to score the symptoms and physical examination results. This system is a simple method to evaluate DN. Easy to use
Table 1: Demographic characteristics of the patients

\begin{tabular}{|c|c|c|}
\hline Variables & Group & Sample (\%) \\
\hline \multirow[t]{3}{*}{ Age (years) } & $50-59$ & $21(35.6)$ \\
\hline & $60-69$ & $19(32.2)$ \\
\hline & $70-80$ & $19(32.2)$ \\
\hline \multirow[t]{2}{*}{ Sex } & Male & $26(44.1)$ \\
\hline & Female & $33(55.9)$ \\
\hline \multirow[t]{4}{*}{ Duration of DM (years) } & $5-9$ & $32(54.2)$ \\
\hline & $10-19$ & $20(33.9)$ \\
\hline & $20-29$ & $5(8.5)$ \\
\hline & $\geq 30$ & $2(3.4)$ \\
\hline \multirow[t]{2}{*}{ Blood glucose } & Normal/controlled & $26(44.1)$ \\
\hline & No & $33(55.9)$ \\
\hline \multirow[t]{2}{*}{ Hypertension } & High blood pressure & $32(54.2)$ \\
\hline & Normotension & $27(45.8)$ \\
\hline \multirow[t]{2}{*}{ Cholesterol } & High total cholesterol & $33(55.9)$ \\
\hline & Normal total cholesterol & $26(44.1)$ \\
\hline \multirow[t]{2}{*}{ Heart disease } & Yes & 7 (11.9) \\
\hline & No & $52(88.1)$ \\
\hline \multirow[t]{2}{*}{ Smoking } & Yes & $11(18.6)$ \\
\hline & No & $48(81.4)$ \\
\hline \multirow[t]{3}{*}{ Body mass index } & Normal & $40(67.8)$ \\
\hline & Over weight & $17(28.8)$ \\
\hline & Obese & $2(3.4)$ \\
\hline
\end{tabular}

DM: Diabetes mellitus

Table 2: Classification of the patients based on neuropathic scores

\begin{tabular}{lll}
\hline Neuropathy & Score & Samples (\%) \\
\hline No neuropathy & $0-5$ & $48(81.4)$ \\
Mild neuropathy & $6-8$ & $9(15.3)$ \\
Moderate neuropathy & $9-11$ & $1(1.7)$ \\
Severe neuropathy & $\geq 12$ & $1(1.7)$ \\
Total samples & & $59(100)$ \\
\hline
\end{tabular}

Table 3: Relationship between neuropathy and risk factors

\begin{tabular}{lll}
\hline Variables & & Significance \\
\cline { 1 - 2 } Dependent & Independent & \\
\hline Neuropathy & Age & 0.857 \\
& Sex & 0.067 \\
& Education & 0.502 \\
& Duration of DM & 0.077 \\
& Blood glucose & 0.532 \\
& Hypertension & 0.416 \\
& Cholesterol & 0.160 \\
& Heart disease & 0.401 \\
& Smoking & 0.087 \\
& Body mass index & 0.765 \\
\hline
\end{tabular}

DM: Diabetes mellitus

and acceptable by the patient, the tool can be used to classify the severity level and correlated with the clinical changes associated with the development of DN. Each patient was asked about the existence of pain sensation (characteristics of neuropathic pain), such as burning and pricked sensation, numbness, tingling, and weakness on the feet. Sensory examination was conducted for feet first and given "normal" or "abnormal" value [16-19].

The examinations include (1) symptoms, foot symptoms (foot pain), numbness, tingling, weakness, ataxia, and upper limp symptoms. Each examination was given a score; $0=$ no symptom, 1 =the symptoms existed, and with maximum score of $6,(2)$ examination of reflex was conducted on both feet for patella and Achilles reflexes; $2=$ no reflex, $0=$ normal reflex, with maximum total score of 8 , (3) sensory examination included pinprick, temperature, light touch, vibration, and position sense; 
Table 4: Sample distribution based on the questionnaire answer

\begin{tabular}{|c|c|c|c|c|c|c|}
\hline \multicolumn{7}{|l|}{ Number of samples with correct answer } \\
\hline \multirow[t]{2}{*}{ Points of question } & \multicolumn{3}{|c|}{ Neuropathy } & \multicolumn{3}{|c|}{ No neuropathy } \\
\hline & SI (\%) & I1 (\%) & I2 (\%) & SI (\%) & I1 (\%) & I2 (\%) \\
\hline General knowledge on diabetic neuropathy & $6(54.5)$ & $7(68.2)$ & $10(90.9)$ & $29(61.5)$ & $31(64.6)$ & $44(90.6)$ \\
\hline Signs and symptoms of diabetic neuropathy & $8(72.7)$ & $9(86.4)$ & $9(86.4)$ & $38(79.2)$ & $41(86.5)$ & 45 (92.7) \\
\hline Age is a risk factor for diabetic neuropathy & $9(82.0)$ & $9(81.8)$ & $9(81.8)$ & 33 (68.8) & $34(70.8)$ & $39(81.3)$ \\
\hline Treatment for diabetic neuropathy & $2(18.2)$ & $4(36.4)$ & $3(27.3)$ & $15(31.3)$ & $22(45.8)$ & $17(35.4)$ \\
\hline Lifestyle of patients with DM & $8(77.3)$ & $9(79.5)$ & $8(77.3)$ & $40(82.8)$ & $41(84.4)$ & $42(87.5)$ \\
\hline Mean \pm standard deviation & $6.6 \pm 2.8$ & $7.6 \pm 2.2$ & $7.8 \pm 2.8$ & $31 \pm 9.9$ & $33.8 \pm 7.9$ & $37.4 \pm 11.6$ \\
\hline
\end{tabular}

SI: Before Intervention, I1: Intervention 1, I2: Intervention 2, Number of samples=59 patients, DM: Diabetes mellitus

$1=$ abnormal, $0=$ normal, and with maximum total score of 19 . Based on the neuropathic scores, the patients were classified as, (1) having no neuropathy with a score of $0-5,(2)$ having mild neuropathy with a score of 6-8, (3) having moderate neuropathy with a score of 9-11, and (4) having severe neuropathy with a score of $\geq 12$ [16-19].

In this study, education was given to all of the respondents, both with and without DN, with the aim of improving their knowledge and motivation. The education included healthy behavioral promotion to obtain an optimum outcome. During the education sessions, the respondents were provided with brochures that contained information on DN and its aspects, including the healthy life behavior. The materials included, (1) healthy eating pattern, (2) the importance of physical exercises, (3) the use of diabetic treatment and drugs for certain situations in a safe and regular way, (4) self-monitoring of glucose level, and (5) routine foot care. The recommended actions to do by the patients included (1) not to walk on bare foot, including on the sand and water, (2) doing foot examination every day and reporting it to the physician when redness, wound, and scaling are identified, (3) making sure that there is no foreign object on the sandals or shoes, (4) always maintaining the feet in clean and dry condition as well as applying moisturizing on the dry feet, (5) regular nail trimming, (6) wearing comfortable socks, (7) make sure that the shoes are not too tight, too loose, or too high, and (8) no to use pillow or a bottle containing hot water or stone for the feet.

There are many risk factors for DN found in this study. The risk factor of age is correlated with blood clotting. There are two factors, which cause blood clotting, namely intrinsic and extrinsic factors. Both factors unite to activate prothrombin to thrombin. Intracellular hyperglycemia leads to the formation of advanced glycation end-product (AGEs). The process originally forms reversible glycation; sustained hyperglycemia will lead to irreversible process that will contribute to the increase of AGEs level in the patients with diabetes mellitus. The AGEs inhibit the production of prostacyclin and induce plasminogen activator inhibitor-1 because of thrombocyte aggregation and fibrin stabilization that facilitate the thrombocytes, microthrombus activated by AGEs, to cause local hypoxia and microangiopathy, and eventually to neuropathy [6]. The risk factors for DN include uncontrolled blood glucose, duration of diabetes mellitus, and cardiovascular diseases (hyperlipidemia, hypertension, smoking, and increased BMI) [5]. Chronic hyperglycemia in the patients with diabetes mellitus leads to endothelial dysfunction, due to the increase in AGEs pathway, polyol pathway, free radicals, and protein kinase $\mathrm{C}$ pathway. Endothelial dysfunction will lead to microangiopathy and nerve hypoxia, and eventually lead to the irreversible damage of nerve structure. Risk factors of endothelial dysfunction include chronic hyperglycemia, elevated fat levels, high blood pressure, elevated BMI, and smoking habit [5].

In this study, many respondents did not have DN since they attempted to improve their knowledge and applied it on their health, compared to those who had DN. It was possible that they had the DN earlier that they had the knowledge since they did not pay attention to the risk factors of DN. Age is another risk factor for the patients with diabetes mellitus to have neuropathy. The research found that $82 \%$ of the respondents had knowledge on the age as a risk factor before the intervention; it remained $82 \%$ after the first intervention and after the second intervention. In the samples without neuropathy, $68.8 \%$ of the respondents provided a correct answer; it increased to $70.8 \%$ after the first intervention and $81.3 \%$ after the second intervention. This shows that the respondent did not have neuropathy because they attempted to improve their knowledge and applied it on their health, compared to those without neuropathy.

When it comes to knowledge about the treatment for DN, $18 \%$ of the respondents with neuropathy provided correct answer before the intervention; it increased to $36.4 \%$ after the first intervention and decreased to $27.3 \%$ after the second intervention. In the samples without DN, 31.3\% provided correct answer before the intervention; it increased to $45.8 \%$ after the first intervention and decreased to $35.4 \%$ after the second intervention. This shows a similar character in that respondents without neuropathy had improving knowledge after each intervention compared to those with DN.

For the risk factor of lifestyle, $77.3 \%$ of the respondents with DN provided correct answer before the intervention; it increased $79.5 \%$ after the first intervention and remained $79 \%$ after the second intervention. In the samples without DN, 83\% of the respondents provided correct answer; it increased to $84.4 \%$ after the first intervention and $87.5 \%$ after the second intervention. In line with the study conducted by Suppapitiporn et al., a good outcome was obtained from the patients who received counseling, compared to those who did not [19].

\section{CONCLUSION}

Based on the research, it can be concluded that from the 59 patients involved in this study, $17.6 \%$ had DN and the remaining $81.4 \%$ did not. Blood glucose, age, duration of disease, hypertension, heart disease, obesity, high cholesterol level, and elevated BMI are some of the risks factors for DN in the patients with diabetes mellitus. Pharmacist intervention showed an increase on the patient's knowledge about DN and also a significant decrease on the patient's blood glucose level $(\mathrm{p}<0.05)$.

\section{REFERENCES}

1. American Diabetes Association. Diagnosis and classification of diabetes mellitus. Diabetes Care 2012;35 Suppl 1:S64-71.

2. King H, Aubert RE, Herman WH. Global burden of diabetes, 1995-2025: Prevalence, numerical estimates, and projections. Diabetes Care 1998;21(9):1414-31.

3. American Diabetes Association. Standards of medical care in diabetes 2013. Diabetes Care 2013;36 Suppl 1:S11-66

4. National Diabetes Information Clearinghouse. National Diabetes Statistics, 2011. National Institute of Diabetes and Digestive and Kidney Diseases (NIDDK); 2011.

5. Tesfaye S. Epidemiology and etiology of diabetic peripheral neuropathies. Adv Stud Med 2004;4(10G):S1014-21.

6. Feldman EL, Vincent A. Review the prevalence, impact, and multifactorial pathogenesis of diabetic peripheral neuropathy. Adv Stud Med 2004;4(8A):S642-9.

7. Morkrid K. The Prevalence of and Risk Factors for Diabetic Peripheral Neuropathy Among Type 2 Diabetic Outpatients in Bangladesh. 
University of Oslo, Faculty of Medicine, Norway. 2007. Available from: https://www.duo.uio.no/bitstream/handle/10852/30151/69091. pdf? sequence $=1$. [Last accessed on 2016 Jan 08]

8. Oguejiofor OC, Odenigbo CU, Oguejiofor CB. Evaluation of the effect of duration of diabetes mellitus on peripheral neuropathy using the United Kingdom screening test scoring system, bio-thessiometry and aesthesiometry. Niger J Clin Pract 2007;13(3):240-7.

9. Cornblath DR. Diabetic neuropathy - diagnostic methods. Adv Stud Med 2004;4:8A.

10. Bril V, Perkins B, Toth C. Neuropathy: Clinical practice guidelines expert committee. Can J Diabetes 2013;37:S142-4.

11. Canadian Diabetes Association. Clinical practice guidelines for the prevention and management of diabetes in Canada. Can J Diabetes 2008;32 Suppl 1:S140-41, S199-201.

12. De Block CE, De Leeuw IH, Van Gaal LF. Impact of overweight on chronic microvascular complications in Type 1 diabetic patients. Diabetes Care 2005;28(7):1649-55

13. Feldman EL. Etiology of diabetic microvascular disease and scientific rationale for new therapeutic targets. Adv Stud Med 2005;5(3A):S138-43.
14. Navale AM, Paranjape AN. Role of inflammation in development of diabetic complications and commonly used inflammatory markers with respect to diabetic complications. Int $\mathrm{J}$ Pharm Pharm Sci 2013;5 Suppl 2:1-15

15. Rampello L, Vecchio I, Malaguarnera G. Diabetic neuropathy diagnosis. Acta Med Mediterr 2012;28:133-8

16. Bril V, Perkins BA. Validation of the Toronto clinical scoring system for diabetic polyneuropathy. Diabetes Care 2002;25(11):2048-52.

17. Tesfaye S, Vileikyte L, Rayman G, Sindrup SH, Perkins BA, Baconja M, et al. Painful diabetic peripheral neuropathy: Consensus recomendations on diagnosis, assesment and management. Diabetes Metab Res Rev 2011;27(7):629-38.

18. Bril V, Tomioka S, Buchanan RA, Perkins BA. Complications reliability and validity of the modified Toronto clinical neuropathy score in diabetic sensorimotor polyneuropathy. Diabet Med 2009;26(3):240-6.

19. Suppapitiporn S, Chindavijak B, Onsanit S. Effect of diabetes drug counseling by pharmacist, diabetic disease booklet and special medication containers on glycemic control of Type 2 diabetes mellitus: A randomized controlled trial. J Med Assoc Thai 2005;88 Suppl 4:S134-41. 